\title{
Methods of Constructing Analytic Functions to Generate Airfoil Profiles
}

\author{
H.B. Jiang, Y.R. Li, Z.Q. Cheng \\ Logistics College \\ Naval University of Engineering \\ Tianjin, China
}

\begin{abstract}
To facilitate the airfoil design, this paper studied the methods of constructing analytic functions to generate airfoil profiles. By Taylor series, Joukowsky airfoil function was simplified to a simple expression, and its coefficients and exponents were redefined as general constants. A series of simple airfoils can be generated by changing the assignment of the constants in the expression. Further, many complex airfoil images could be obtained by recombining upper profile functions and lower profile functions of other airfoils or by adding a number of thickness functions in the expression. Studies have shown that the structure of the expression is simple and the geometric meaning of the parameters in it is clear, which is suited for expressing airfoil shape; according to the parameter assignment rule summarized in this paper, the desired airfoil shape can be easily generated.
\end{abstract}

Keywords- airfoil design; airfoil profile; medial-camber line; thickness function; analytic method; parameter expressions

\section{INTRODUCTION}

The shape of airfoil determined pressure distribution of the flow field and the performance of the airfoil, which is the basis for the analysis of flow, so the airfoil design, especially the method to generate or express shape of the airfoil is very important. Airfoil profile is typically determined by the coordinate database. An airfoil profile could be obtained by sequentially connecting the data image with a smooth curve. Therefore a set of coordinate data only represents one airfoil.

In addition, the airfoil geometry shape is also described using a variety of methods, mainly there are the shape parameterization method, the shape function perturbation method and analytic function method. The shape parameterization method describes the sizes of various parts of the airfoil by using several parameters which have clear meaning, but does not give the analytical expression $[1,2]$. Shape function perturbation method determines the shape by adding perturbation function to the original airfoil shape function, and the Hicks-Henne function [3] was more used as the perturbation function. But this method is highly dependent on the original airfoil, if original airfoil shape is not smooth, then the shape of the airfoil of design is not smooth, and may directly affect the smoothness of the pressure coefficient curve. In addition, this method can not change the initial geometric parameters of the leading edge and trailing edge angles of the original airfoil [4, 5]. Analytic function method is to use analytic functions to express airfoil profiles, such as the polynomial expression of 4,5 -digit NACA airfoil series in the early and the series expression method in the recent period [6]. Because the parameter changes have a huge impact on the global shape of the airfoil and fine-tuning effect is not good, these methods are more suitable for static airfoil.

This paper intends to explore a method to generate airfoil profiles with analytic functions, with which a bunch of new airfoil can be generated when the parameter values in the functions were changed. The geometric meaning of the function itself and all the parameters is very clear, so we can achieve reverse design to generate airfoil through fine-tuning parameter values according to the expected direction. In addition, the pressure distribution and lift performance can be calculated with analytical method by using of the function expression of the airfoil [7], which can greatly simplify the design process, significantly improve design efficiency.

\section{CONSTRUCTING AIRFOIL PROFILE FUNCTION}

Joukowsky airfoil profile expression is [8]

$$
y=\sqrt{\frac{1}{4}+\frac{1}{64 \varepsilon^{2}}-x_{C}^{2}}-\frac{1}{8 \varepsilon} \pm \frac{2 \delta}{3 \sqrt{3}}\left(1-2 x_{C}\right) \sqrt{1-4 x_{C}^{2}}
$$

Where $x$ is abscissa (chord coincident with the axis of abscissa), $y$ is ordinate, $\delta$ is the maximum thickness, $\varepsilon$ is the maximum camber. Dimensionless parameters are above one. The formula (1) expresses upper profile if with positive sign, and lower profile if with negative sign. The chord length in the formula is one.

Camber is very small relative to the chord length, so the simplification formula by Taylor series expansion with ignoring three times or more order of camber was obtained:

$$
y=\varepsilon\left(1+2 x_{C}\right)^{1}\left(1-2 x_{C}\right)^{1} \pm \frac{2 \delta}{3 \sqrt{3}}\left(1+2 x_{C}\right)^{\frac{1}{2}}\left(1-2 x_{C}\right)^{\frac{3}{2}}
$$

This is the airfoil expression that the origin locates at the chord midpoint. The first term in the expression is medialcamber line, and its maximum is $\varepsilon$, which is the airfoil camber. The second term represents the thickness of the airfoil. It is easy to prove that the maximum distance between the upper profile and the lower profile is $\delta$. Let $1+2 x_{C}=2 x$, Then $1-2 x_{C}=$ 2(1-x), Therefore

$$
y=2 \varepsilon x^{1}(1-x)^{1} \pm 1.54 \delta x^{\frac{1}{2}}(1-x)^{\frac{3}{2}}
$$


This is the airfoil expression that the origin locates at the front edge point.

In the function expression (3), the coefficients and exponents (are called parameters in this paper) have a significant effect on the image shape of the function. To construct the general function of airfoil profile, the coefficients and exponents in expression (3) could be given a patulous definition, so the general function of airfoil profile similar to the expression (3) could be obtained:

$$
y=p x^{a}(1-x)^{b} \pm q x^{c}(1-x)^{d}
$$

Where $p, a, b, q, c$ and $d$ are constants greater than 0 . With expression (4), a series of airfoils with different shapes could be generated by assign different values to the constants (parameters); Joukowsky airfoil profile function can be considered a special example of this expression. In expression (4), the size of $p$ is proportional to the camber of the airfoil, for example, it represents symmetric airfoil when $p=0$ or Joukowsky airfoil when $a=b=d=1$; It also can generate front bend airfoil when $a$ is far greater than $b$ or rear bend airfoil when $b$ is far greater than $a$. Figure 1 shows several examples of airfoils generated with different parameter values, and the solid curve in the Figure 1 is the medial-camber line.

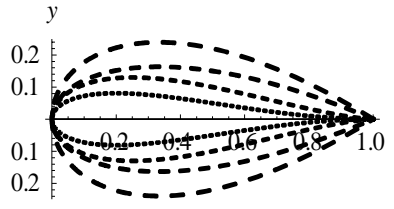

(a)Symmetrical airfoils.

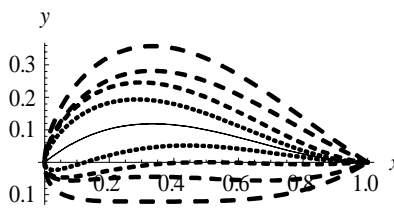

(c) Front bend airfoils

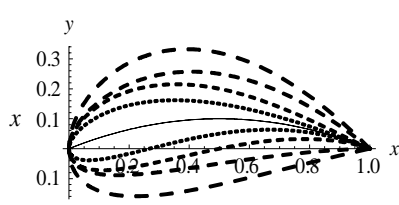

(b)Joukowsky airfoils

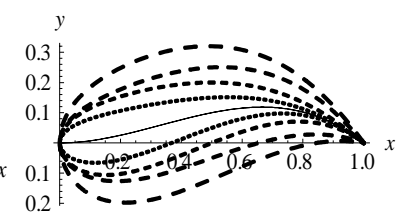

(d) Rear bend airfoils
FIGURE I. AIRFOIL EXAMPLES GENERATED BY FUNCTION EXPRESSIONS.

\section{THE AFFECTS OF THE PARAMETERS TO THE SHAPE OF AIRFOIL}

In expression (4), the value of $x$ is between 0 and $1 / 2$ in the front, so its exponents ( $a$ or $c$ ) have great influence on the forepart shape of airfoil, and similarly the value of $(1-x)$ is between 0 and $1 / 2$ in the back part $(1 / 2<x<1)$, so its exponents ( $b$ or $d$ ) have great influence on the back part shape.

In the expression, the first term represents the medialcamber line, and there are three parameters to control airfoil bend shape: coefficient $p$ controls the overall height of the medial-camber line (the larger the higher), the exponent $a$ mainly controls the height of the forepart of the medial-camber line (the larger the lower), and the exponent $b$ mainly controls the height of the back part of the medial-camber line (the larger the lower); The second term represents thickness of airfoil, and there are three parameters to control airfoil thickness shape: the coefficient $q$ controls overall thickness trend (the greater the thicker), the exponent $c$ mainly controls forepart thickness (the greater the thinner), the exponent $d$ mainly controls back part thickness (the greater the thinner). The trend of influence of airfoil parameter value changes was shown in Table 1.

TABLE I. THE AIRFOIL SHAPE CHANGE TRENDS WITH THE PARAMETER VALUE CHANGES.

\begin{tabular}{|c|c|c|c|}
\hline \multicolumn{2}{|c|}{ Parameters } & $\begin{array}{c}\text { When the parameters } \\
\text { become larger }\end{array}$ & $\begin{array}{c}\text { When the parameters } \\
\text { become smaller }\end{array}$ \\
\hline \multirow{3}{*}{$\begin{array}{l}\text { Parameters of } \\
\text { medial-camber } \\
\text { line function }\end{array}$} & $p$ & $\begin{array}{c}\text { Overall shape becomes } \\
\text { higher }\end{array}$ & $\begin{array}{c}\text { Overall shape becomes } \\
\text { lower }\end{array}$ \\
\hline & $a$ & $\begin{array}{c}\text { Forepart becomes lower } \\
\text { significantly; } \\
\text { Back part becomes lower } \\
\text { slightly }\end{array}$ & $\begin{array}{c}\text { Forepart becomes higher } \\
\text { significantly; } \\
\text { Back part becomes } \\
\text { higher slightly }\end{array}$ \\
\hline & $b$ & \begin{tabular}{|c|} 
Back part becomes lower \\
significantly; \\
Forepart part becomes \\
lower slightly \\
\end{tabular} & $\begin{array}{c}\text { Back part becomes } \\
\text { higher significantly; } \\
\text { Forepart becomes higher } \\
\text { slightly }\end{array}$ \\
\hline \multirow{3}{*}{$\begin{array}{l}\text { Parameters of } \\
\text { thickness } \\
\text { function }\end{array}$} & $q$ & \begin{tabular}{|c|}
$\begin{array}{c}\text { Overall shape becomes } \\
\text { thicker }\end{array}$ \\
\end{tabular} & \begin{tabular}{|c|}
$\begin{array}{c}\text { Overall shape becomes } \\
\text { thinner }\end{array}$ \\
\end{tabular} \\
\hline & $c$ & $\begin{array}{c}\text { Forepart becomes thinner } \\
\text { significantly; } \\
\text { Back part becomes } \\
\text { thinner slightly }\end{array}$ & $\begin{array}{c}\text { Forepart becomes thicker } \\
\text { significantly; } \\
\text { Back part becomes } \\
\text { thicker slightly }\end{array}$ \\
\hline & $d$ & \begin{tabular}{|c|} 
Back part becomes \\
thinner significantly; \\
Forepart becomes thinner \\
slightly
\end{tabular} & $\begin{array}{c}\text { Back part becomes } \\
\text { thicker significantly; } \\
\text { Forepart becomes thicker } \\
\text { slightly }\end{array}$ \\
\hline
\end{tabular}

A simple rule could be summed from the table: the larger the coefficients become, the higher and the thicker the overall shape becomes (with the same trend); The Larger the exponents become, the lower and the thinner the shape becomes (with the opposite trend); The exponents of $x$ have significantly affect to the forepart shape, and the exponents of (1-x) have significantly affect to the back part shape. Mastered this simple rule we will facilitate the assignment of the parameter values in order to generate the desired shape of the airfoil, or approach to existing airfoil shape.

\section{THE FUNCTION EXPRESSIONS OF COMPLEX AIRFOIL PROFILES}

In order to express the airfoil which trailing edge has a smooth shape, and facilitate fine-tuning airfoil shape, one or more thickness items could be added on the formula (4); To produce complex airfoil structures, the upper profile and the lower profile of different airfoils could be recombined to generate a new airfoil (i.e. different values could be sign to the parameters of the upper profile and the lower profile formula). The general expressions of airfoil profiles according to those rules could be obtained:

$$
\left\{\begin{array}{l}
y_{u}=p_{u} x^{a_{u}}(1-x)^{b_{u}}+\sum_{i=1}^{M} q_{u i} x^{c_{u i}}(1-x)^{d_{u i}} \\
y_{l}=p_{l} x^{a_{l}}(1-x)^{b_{l}}-\sum_{j=1}^{N} q_{l j} x^{c_{i j}}(1-x)^{d_{l j}}
\end{array}\right.
$$

Where $x, y$ represent the horizontal and vertical coordinates; subscript $u, l$ represent the upper profile and the lower profile of airfoil, the values of the upper profile parameters $p_{u}, a_{u}, b_{u}$, $q_{u i}, c_{u i}, d_{u i}(i=1,2,3, \ldots, M)$ and the lower profile parameters $p_{l}, a_{l}$, 
$b_{l}, p_{l j}, a_{l j}, b_{l j}(j=1,2,3, \ldots, N)$ are constant greater than zero, $M$ and $N$ are positive integers.

When $M=N=2$, the expressions of airfoil profiles are

$$
\left\{\begin{array}{l}
y_{u}=p_{u} x^{a_{u}}(1-x)^{b_{u}}+q_{u} x^{c_{u}}(1-x)^{d_{u}}+r_{u} x^{e_{u}}(1-x)^{f_{u}} \\
y_{l}=p_{l} x^{a_{l}}(1-x)^{b_{l}}-q_{l} x^{c_{l}}(1-x)^{d_{l}}-r_{l} x^{e_{l}}(1-x)^{f_{l}}
\end{array}\right.
$$

If $M=N=1$ and the corresponding parameter values in the upper profile and the lower profile are the same, the airfoil function expression will be in complete agreement to formula (4).

\section{THE EXAMPLES TO EXPRESS EXISTING AIRFOIL}

In the design of airfoil, some existing airfoils were usually referred to generate new airfoils through a variety of methods to fine-tune the airfoil shape and compute the airfoil performances under constraints. As an example, some analytic functions will be discussed to express existing wind turbine airfoils approximately.

Airfoil profiles are generally described by the coordinate database. It is easy to draw a point image of coordinate data and generate airfoil image by connecting all points with a smooth curve sequentially. With function curve to approximate point images, the easiest method is based on the difference between the points and the curve to gradually adjust the values of the parameters in the function according to above simple rule. Since the geometric meaning of the parameters in the functions given in this paper is clear (see the Table), the approaching process is simple, and generally a function expression and its graphic could be obtained just a few minutes.

Here two examples of the wind turbine airfoils with distinct characteristics were shown in Figure2 and Figure3, which are DU 91-W2-250 airfoil from Netherlands, and FFAW3-301 airfoil from Sweden.

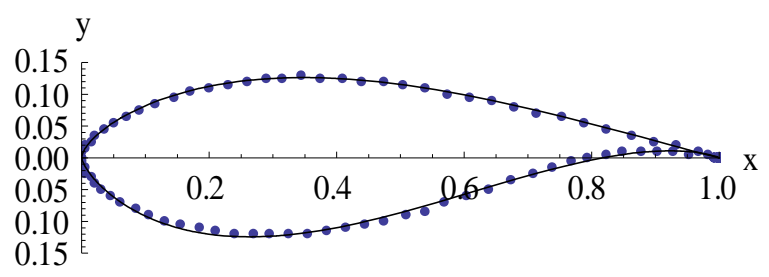

FIGURE II. DU 91-W2-250 AIRFOIL APPROACHING CURVES.

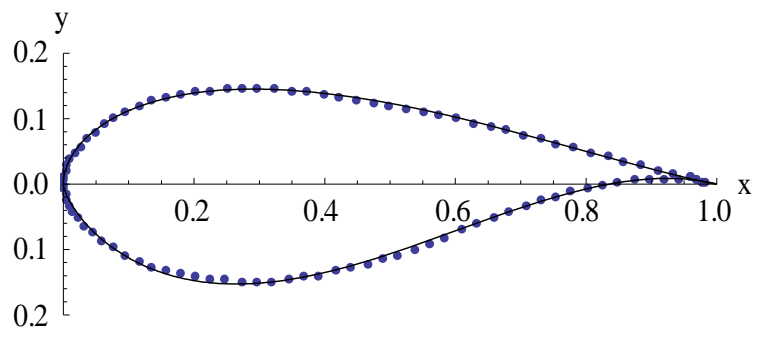

FIGURE III. FFA-W3-301 AIRFOIL APPROACHING CURVES.

The fitting curves to the coordinate data points were generated by several analytic functions, which are
DU 91-W2-250 airfoil

$$
\left\{\begin{array}{l}
y_{u}=0.2 x^{1.1}(1-x)^{2.3}+0.295 x^{0.58}(1-x)^{1.03} \\
y_{l}=0.26 x^{0.75}(1-x)^{0.9}-0.85 x^{0.73}(1-x)^{1.6}
\end{array}\right.
$$

FFA-W3-301 airfoil

$$
\left\{\begin{array}{l}
y_{u}=0.2 x^{0.61}(1-x)^{1.6}+0.24 x^{0.48}(1-x)^{1.1} \\
y_{l}=0.27 x^{2.6}(1-x)^{1}-0.68 x^{0.7}(1-x)^{1.7}
\end{array}\right.
$$

Similarly, the function expressions of other airfoils could be obtained, and further, a series of new airfoils could be generated by assigning the different values to the parameters with fine-tuning the airfoil shape in design.

\section{CONCLUSIONS}

(1) Joukowsky airfoil profile expression can be simplified to the analytic functions expressed by medial-camber line and thickness formulas, which are simple in structure, clear in geometric meaning of the parameters, and quite suited for the expression of the airfoil shape.

(2) The airfoil with smooth trailing edge shape could be obtained by the way of increasing thickness function items.

(3) According to the assignment rules to parameters in airfoil functions, the expected shape of airfoil could be generated easily, and the shape of existing airfoil could be approached.

\section{ACKNOWLEDGEMENT}

This research was financially supported by the National Natural Science Foundation of China (Grant No. 51375489).

\section{REFERENCES}

[1] T Ray, H M Tsai. Swarm algorithm for single and multiobjective airfoil design optimization. AIAA Journal, 42(2), pp.366-373, 2004.

[2] H Sobieczky, Parametric Airfoils and Wings. Note on Numerical Fluid Mechanics, 68, pp. 71-88,1998.

[3] Hicks R, Henne P. Wing Design by Numerical optimization. Aircraft, 15(7), pp. 407-413, 1978.

[4] LIU Zhou, ZHU Zi-qiang, FU Hong-yan, et al. Design of airfoil with high ratio of lift over drag. Acta Aerodynamica Sinica, 22(4), pp. 410415, 2004 (in Chinese).

[5] CHEN Jin, ZHANG Shiqiang, EECEN P J, et al. Parametric Representation and Convergence of Wind Turbine Airfoils. Journal of Mechanical Engineering, 46(10), pp. 132-138, 2010.(in Chinese).

[6] CHEN Jin, ZHANG Shiqiang, PANG Xiaoping, et al. Method for designing special wind turbine airfoil profile and special wind turbine airfoil profile,.China, 200910191275, 2012. (in Chinese).

[7] Haibo Jiang, Zhongqing Cheng, Yunpeng Zhao. Function airfoil and its pressure distribution and lift coefficient calculation. Applied Mechanics and Materials, 328, pp. 351- 356, 2013.

[8] Dong ZN, Zhang ZX. Incompressible Fluid Mechanics. Beijing: Tsinghua University Press, pp. 264-266, 2003. (in Chinese). 